\title{
Existence of Solutions to the System of Generalized Implicit Vector Quasivariational Inequality Problems
}

\author{
Zhi Lin \\ College of Science, Chongqing Jiaotong University, Chongqing 400074, China \\ Correspondence should be addressed to Zhi Lin, linzhi7525@163.com \\ Received 31 March 2009; Revised 30 July 2009; Accepted 3 September 2009 \\ Recommended by Yeol Je Cho \\ We study the system of generalized implicit vector quasivariational inequality problems and prove \\ a new existence result of its solutions by Kakutani-Fan-Glicksberg's fixed points theorem. As a \\ special case, we also derive a new existence result of solutions to the generalized implicit vector \\ quasivariational inequality problems.
}

Copyright (c) 2009 Zhi Lin. This is an open access article distributed under the Creative Commons Attribution License, which permits unrestricted use, distribution, and reproduction in any medium, provided the original work is properly cited.

\section{Introduction}

The system of generalized implicit vector quasivariational inequality problems generalizes the generalized implicit vector quasivariational inequality problems, and the latter had been studied in [1-3]. In this paper, we study the system of generalized implicit vector quasivariational inequality problems and prove a new existence result of its solutions by Kakutani-Fan-Glicksberg's fixed points theorem. For other existence results with respect to the system of generalized implicit vector quasivariational inequality problems, we refer the reader to [4-6] and references therein.

Let $I$ be an index set (finite or infinite). For each $i \in I$, let $X_{i}$ and $Y_{i}$ be two Hausdorff topological vector spaces, $K_{i}$ a nonempty subset of $X_{i}$, and $C_{i}$ a closed, convex and pointed cone of $Y_{i}$ with int $C_{i} \neq \emptyset$, where int $C_{i}$ denotes the interior of $C_{i}$. Denote that $K_{\hat{i}}=\prod_{j \in I, j \neq i} K_{j}, K=\prod_{i \in I} K_{i}=K_{i} \times K_{\hat{i}}, X=\prod_{i \in I} X_{i}$. For each $x \in K$, we can write $x=\left(x_{i}, x_{\hat{i}}\right)$. For each $i \in I$, let $D_{i}$ be a nonempty subset of the continuous linear operators space $L\left(X_{i}, Y_{i}\right)$ from $X_{i}$ into $Y_{i}$ and let $F: D_{i} \times K_{i} \times K_{i} \longrightarrow Y_{i}, G_{i}: K \longrightarrow 2^{K_{i}}, T_{i}: K \longrightarrow 2^{D_{i}}$ be three set-valued maps, where $2^{D_{i}}$ and $2^{K_{i}}$ denote the family of all nonempty subsets of $D_{i}$ and $K_{i}$, respectively. The system of generalized implicit vector quasivariational inequality problems 
(briefly, SGIVQIP) is as follows: find $\bar{x}=\left(\bar{x}_{i}, \bar{x}_{\bar{i}}\right) \in K$ such that for each $i \in I, \bar{x}_{i} \in G_{i}(\bar{x})$, and

$$
\forall y_{i} \in G_{i}(\bar{x}), \exists \bar{u}_{i} \in T_{i}(\bar{x}), \quad F_{i}\left(\bar{u}_{i}, \bar{x}_{i}, y_{i}\right) \not \subset-\operatorname{int} C_{i} .
$$

$\bar{x}=\left(\bar{x}_{i}, \bar{x}_{\bar{i}}\right)$ is said to be a solution of the SGIVQIP. An SGIVQIP is usually denoted by $\left\{K_{i}, D_{i}, G_{i}, T_{i}, F_{i}\right\}_{i \in I}$.

If $I$ is a singleton, then the SGIVQIP coincides with the generalized implicit vector quasivariational inequality problems (briefly, GIVQIP). A GIVQIP is usually denoted by $\{K, D, G, T, F\}$.

Throughout this paper, unless otherwise specified, assume that for each $i \in I, K_{i}$ is a nonempty convex compact subset of a Banach space $X_{i}, Y_{i}$ is a Hausdorff topological vector space, and $C_{i}$ is a closed, convex, and pointed cone of $Y_{i}$ with int $C_{i} \neq \emptyset$, where int $C_{i}$ denotes the interior of $C_{i}$.

\section{Preliminaries}

In this section, we introduce some useful notations and results.

Definition 2.1. Let $X$ and $Y$ be two topological spaces and $K$ a nonempty convex subset of $X$. $F: K \longrightarrow 2^{\Upsilon}$ is a set-valued map.

(1) $F$ is called upper semicontinuous at $x_{0} \in K$ if, for any open set $G \supset F\left(x_{0}\right)$, there exists an open neighborhood $U$ of $x_{0}$ in $K$ such that for all $x \in U$,

$$
G \supset F(x)
$$

and upper semicontinuous on $K$ if it is upper semicontinuous at every point of $K$.

(2) $F$ is called lower semicontinuous at $x_{0} \in K$ if, for any open set $G \cap F\left(x_{0}\right) \neq \emptyset$, there exists an open neighborhood $U$ of $x_{0}$ in $K$ such that for all $x \in U$,

$$
G \cap F(x) \neq \emptyset
$$

and lower semicontinuous on $K$ if it is lower semicontinuous at every point of $K$.

(3) $F$ is called continuous at $x_{0} \in K$ if, it is both upper semicontinuous and lower semicontinuous at $x_{0}$; and continuous on $K$ if it is continuous at every point of $K$.

Definition 2.2. Let $X$ and $Y$ be two topological vector spaces and $K$ a nonempty convex subset of $X$.Also $F: K \longrightarrow 2^{Y}$ is a set-valued map.

(1) $F$ is called upper $C$-semicontinuous at $x_{0} \in K$ if, for any open neighborhood $V$ of the zero element $\theta$ in $Y$, there exists an open neighborhood $U$ of $x_{0}$ in $K$ such that, for all $x \in U$,

$$
F(x) \subset F\left(x_{0}\right)+V+C
$$

and upper $C$-semicontinuous on $K$ if it is upper $C$-semicontinuous at every point of $K$. 
(2) $F$ is called lower $C$-semicontinuous at $x_{0} \in K$ if, for any open neighborhood $V$ of the zero element $\theta$ in $Y$, there exists an open neighborhood $U$ of $x_{0}$ in $K$ such that, for all $x \in U$,

$$
F(x) \cap\left(F\left(x_{0}\right)+V+C\right) \neq \emptyset
$$

and lower $C$-semicontinuous on $K$ if it is lower $C$-semicontinuous at every point of K.

(3) $F$ is called $C$-continuous at $x_{0} \in K$ if it is upper $C$-semicontinuous and lower $C$ semicontinuous at $x_{0} \in K$; and $C$-continuous on $K$ if it is $C$-continuous at every point of $K$.

Definition 2.3. Let $X$ and $Y$ be two topological vector spaces and $K$ a nonempty convex subset of $X$. Let $F: K \rightarrow 2^{Y}$ be a set-valued map.

(1) $F$ is called $C$-convex if, for each $x_{1}, x_{2} \in K, t \in[0,1]$,

$$
F\left(t x_{1}+(1-t) x_{2}\right) \subset\left[t F\left(x_{1}\right)+(1-t) F\left(x_{2}\right)\right]-C ;
$$

and $C$-concave if $-F$ is $C$-convex.

(2) $F$ is called $C$-quasiconvex-like if, for each $x_{1}, x_{2} \in K, t \in[0,1]$,

either $F\left(t x_{1}+(1-t) x_{2}\right) \subset F\left(x_{1}\right)-C$ or $F\left(t x_{1}+(1-t) x_{2}\right) \subset F\left(x_{2}\right)-C$;

and $C$-quasiconcave-like if $-F$ is $C$-quasiconvex-like.

Lemma 2.4 ([7, Theorem 1]). Let $K$ be a nonempty paracompact subset of a Hausdorff topological space $X$ and, $Z$ be a nonempty subset of a Hausdorff topological vector space $Y$. Suppose that $S, T$ : $K \mapsto 2^{Z}$ be two set-valued maps with following conditions:

(1) for each $x \in K, \operatorname{coS}(x) \subset T(x)$;

(2) for each $y \in Z, S^{-1}(y)=\{x \in K: y \in S(x)\}$ is open.

Then Thas a continuous selection, that is, there is a continuous map $f: K \mapsto Z$ such that $f(x) \in T(x)$ for each $x \in K$.

\section{Existence of Solutions to the SGIVQIP}

Lemma 3.1. Let $D, W, X$ be three Hausdorff topological spaces, $Z$ a topological vector space, and $C$ a closed, convex, and pointed cone of $Z$. Let $T: W \times X \mapsto 2^{D}$ and $F: D \times W \times W \mapsto 2^{Z}$ be two set-valued maps. Assume that $(w, x, y) \in W \times X \times W$ and

(1) $T(\cdot, \cdot)$ is upper semicontinuous on $W \times X$ with nonempty and compact values;

(2) $F(\cdot, \cdot, \cdot)$ is upper $C$-semicontinuous on $D \times W \times W$ with nonempty and compact values;

(3) for each $u \in T(w, x), F(u, w, y) \subset-\operatorname{int} C$. 
Then there exist open neighborhood $U(w)$ of $w$ and open neighborhood $U(x)$ of $x$, and open neighborhood $U(y)$ of $y$ such that $\left\{F\left(u, w^{\prime}, y^{\prime}\right): u \in T\left(w^{\prime}, x^{\prime}\right)\right\} \subset-\operatorname{int} C$ whenever $w^{\prime} \in U(w)$, $x^{\prime} \in U(x), y^{\prime} \in U(y)$.

Proof. By (3) and compactness of $F(u, w, y)$, there exists an open neighborhood $V(u)$ of the zero element $\theta$ of $Z$ such that $F(u, w, y)+V(u) \subset-\operatorname{int} C$. By (2), there exist open neighborhood $O(u)$ of $u$ and open neighborhood $O_{u}(w)$ of $w$, open neighborhood $O_{u}(y)$ of $y$ such that $F\left(u^{\prime}, w^{\prime}, y^{\prime}\right) \subset F(u, w, y)+V(u)-C \subset-\operatorname{int} C-C \subset-\operatorname{int} C$ whenever $u^{\prime} \in O(u), w^{\prime} \in O_{u}(w), y^{\prime} \in$ $O_{u}(y)$. Since $T(w, x)$ is compact and $\cup_{u \in T(w, x)} O(u) \supset T(w, x)$, there exist finite $u^{1}, u^{2}, \ldots, u^{M} \in$ $T(w, x)$ such that $\bigcup_{j=1}^{M} O\left(u^{j}\right) \supset T(w, x)$. Taking

$$
O(w)=\cap_{j=1}^{M} O_{u^{j}}(w), \quad U(y)=\cap_{j=1}^{M} O_{u^{j}}(y)
$$

Clearly, $O(w)$ and $U(y)$ are open neighborhood of $w$ and $y$, respectively. Thus for each $u \in$ $\cup_{j=1}^{M} O\left(u^{j}\right)$, we have $F\left(u, w^{\prime}, y^{\prime}\right) \subset-\operatorname{int} C$ whenever $w^{\prime} \in O(w), y^{\prime} \in U(y)$. By (1), there exist open neighborhood $U(w)$ of $w$ with $U(w) \subset O(w)$ and open neighborhood $U(x)$ of $x$ such that $T\left(w^{\prime}, x^{\prime}\right) \subset \cup_{j=1}^{M} O\left(u^{j}\right)$ whenever $w^{\prime} \in U(w), x^{\prime} \in U(x)$, which implies that

$$
\left\{F\left(u, w^{\prime}, y^{\prime}\right): u \in T\left(w^{\prime}, x^{\prime}\right)\right\} \subset\left\{F\left(u, w^{\prime}, y^{\prime}\right): u \in \cup_{j=1}^{M} O\left(u^{j}\right)\right\} \subset-\operatorname{int} C .
$$

whenever $w^{\prime} \in U(w), x^{\prime} \in U(x), y^{\prime} \in U(y)$.

The proof is finished.

By Lemma 3.1, we obtain the following result.

Theorem 3.2. Consider an SGIVQIP $\left\{K_{i}, D_{i}, G_{i}, T_{i}, F_{i}\right\}_{i \in I}$. For each $i \in I$, assume that

(1) $G_{i}(\cdot)$ is continuous on $K$ with convex compact values and for each $x \in K$, int $G_{i}(x) \neq \emptyset$;

(2) $T_{i}(\cdot)$ is upper semicontinuous on $K$ with nonempty and compact values;

(3) $F_{i}(\cdot, \cdot, \cdot)$ is upper $C_{i}$ - semicontinuous on $D_{i} \times K_{i} \times K_{i}$ with nonempty and compact values;

(4) for each $x \in K$ and each $u_{i} \in T_{i}(x), F_{i}\left(u_{i}, x_{i}, \cdot\right)$ is $C_{i}$ - convex or $C_{i}$ - quasiconvex-like;

(5) for each $x \in K$ and each $u_{i} \in T_{i}(x)$, if $x_{i} \in \operatorname{int} G_{i}(x)$, then $F_{i}\left(u_{i}, x_{i}, x_{i}\right) \not \subset-i n t C_{i}$, where $x_{i}$ is the ith component of $x$.

Then the SGIVQIP has a solution, that is, there exists $\bar{x}=\left(\bar{x}_{i}, \bar{x}_{\bar{i}}\right) \in K$ such that for each $i \in I, \bar{x}_{i} \in G_{i}(\bar{x})$, and

$$
\forall y_{i} \in G_{i}(\bar{x}), \exists \bar{u}_{i} \in T_{i}(\bar{x}), \quad F_{i}\left(\bar{u}_{i}, \bar{x}_{i}, y_{i}\right) \not \subset-\operatorname{int} C_{i} .
$$


Proof. For each $i \in I$, define a set-valued map $S_{i}: K \rightarrow 2^{K_{i}} \cup\{\emptyset\}$ by

$$
S_{i}(x)=\left\{y_{i} \in K_{i}: F_{i}\left(u_{i}, x_{i}, y_{i}\right) \subset-\operatorname{int} C_{i}, \forall u_{i} \in T_{i}(x)\right\}
$$

Step 1. We prove that the set $J_{i}=\left\{x \in K: G_{i}(x) \cap S_{i}(x)=\emptyset\right\}$ is closed. For any sequence $x^{n} \in J_{i}=\left\{x \in K: G_{i}(x) \cap S_{i}(x)=\emptyset\right\}$ with $x^{n} \rightarrow x^{0}$, we have

$$
\forall y_{i}^{n} \in G_{i}\left(x^{n}\right), \exists u_{i}^{n} \in T_{i}\left(x^{n}\right), \quad F_{i}\left(u_{i}^{n}, x_{i}^{n}, y_{i}^{n}\right) \not \subset-\operatorname{int} C_{i}
$$

If $x^{0} \notin J_{i}$, then there exists $z_{i}^{0} \in G_{i}\left(x^{0}\right)$ such that for each $u_{i} \in T_{i}\left(x^{0}\right), F_{i}\left(u_{i}, x_{i}^{0}, z_{i}^{0}\right) \subset-\operatorname{int} C_{i}$. By Lemma 3.1, there exist open neighborhood $U\left(x^{0}\right)$ of $x^{0}$ and open neighborhood $U\left(z_{i}^{0}\right)$ of $z_{i}^{0}$, such that $\left\{F\left(u_{i}, x_{i}^{\prime}, z_{i}^{\prime}\right): u_{i} \in T\left(x^{\prime}\right)\right\} \subset-\operatorname{int} C_{i}$ whenever $x^{\prime} \in U\left(x^{0}\right), z_{i}^{\prime} \in U\left(z_{i}^{0}\right)$. By (1), there exist $z_{i}^{n} \in G_{i}\left(x^{n}\right)$ such that $z_{i}^{n} \rightarrow z_{i}^{0}(n \rightarrow+\infty)$, which implies that there exists a positive integer $N$ such that $x^{n} \in U\left(x^{0}\right), z_{i}^{n} \in U\left(z_{i}^{0}\right)$ whenever $n>N$. Thus we have $F_{i}\left(u_{i}, x^{n}, z_{i}^{n}\right) \subset-\operatorname{int} C_{i}$, for all $u_{i} \in T_{i}\left(x^{n}\right)$ whenever $n>N$, a contradiction. This shows that $J_{i}$ is closed, that is, $W_{i}=\left\{x \in K: G_{i}(x) \cap S_{i}(x) \neq \emptyset\right\}$ is open.

Without loss of generality, assume that $W_{i} \neq \emptyset$.

Define a set-valued map $P_{i}: K \mapsto 2^{K_{i}} \cup\{\emptyset\}$ by

$$
P_{i}(x)=\operatorname{int} G_{i}(x) \cap S_{i}(x) \text { for each } x \in K \text {. }
$$

Step 2. We prove that for each $x \in W_{i}, P_{i}(x)$ is nonempty and convex.

For each $y_{i} \in S_{i}(x)$, we have $F_{i}\left(u_{i}, x_{i}, y_{i}\right) \subset-\operatorname{int} C_{i}$, for all $u_{i} \in T_{i}(x)$. By Lemma 3.1, there exists an open neighborhood $U\left(y_{i}\right)$ of $y_{i}$ such that $\left\{F_{i}\left(u_{i}, x_{i}, y_{i}^{\prime}\right): u_{i} \in T_{i}(x)\right\} \subset-\operatorname{int} C_{i}$ whenever $y_{i}^{\prime} \in U\left(y_{i}\right)$, which implies that $U\left(y_{i}\right) \subset S_{i}(x)$, that is, $S_{i}(x)$ is open. By (4), it is easy to verify that $S_{i}(x)$ is convex.

Since $G_{i}(x)$ is convex and int $G_{i}(x) \neq \emptyset$, then for each $x \in W_{i}, P_{i}(x)$ is nonempty and convex.

Step 3. We prove that $\left.P_{i}\right|_{W_{i}}$ has a continuous selection $f_{i}: W_{i} \mapsto 2^{K_{i}}$.

For each $y_{i}^{0} \in P_{i}(x)$, we have $y_{i}^{0} \in \operatorname{int} G_{i}(x)$ and $y_{i}^{0} \in S_{i}(x)$. By $y_{i}^{0} \in \operatorname{int} G_{i}(x)$, there exists $\varepsilon_{0}>0$ such that $y_{i}^{0}+\varepsilon_{0} \subset$ int $G_{i}(x)$, where $y_{i}^{0}+\varepsilon_{0}=\left\{z_{i} \in K_{i}: d_{i}\left(z_{i}, y_{i}^{0}\right)<\varepsilon_{0}\right\}$. Since $G_{i}(x)$ is continuous with convex compact values, then there exists an open neighborhood $O(x)$ of $x$ such that

$$
G_{i}(x) \subset G_{i}\left(x^{\prime}\right)+\frac{1}{2} \varepsilon_{0}
$$

whenever $x^{\prime} \in O(x)$, where $G_{i}\left(x^{\prime}\right)+(1 / 2) \varepsilon_{0}=\left\{z_{i} \in K_{i}: d_{i}\left(z_{i}, G_{i}\left(x^{\prime}\right)\right)<(1 / 2) \varepsilon_{0}\right\}$. Thus $y_{i}^{0}+\varepsilon_{0} \subset \operatorname{int} G_{i}(x) \subset G_{i}(x) \subset G_{i}\left(x^{\prime}\right)+(1 / 2) \varepsilon_{0}$ whenever $x^{\prime} \in O(x)$, which implies that $y_{i}^{0}+(1 / 2) \varepsilon_{0} \subset G_{i}\left(x^{\prime}\right)$ whenever $x^{\prime} \in O(x)$, that is, $y_{i}^{0} \in \operatorname{int} G_{i}\left(x^{\prime}\right)$ whenever $x^{\prime} \in O(x)$. This shows that the set $\left\{x \in K: y_{i}^{0} \in \operatorname{int} G_{i}(x)\right\}$ is open. By $y_{i}^{0} \in S_{i}(x)$, we have $F_{i}\left(u_{i}, x_{i}, y_{i}^{0}\right) \subset$ $-\operatorname{int} C_{i}, \forall u_{i} \in T_{i}(x)$. By Lemma 3.1, there exists an open neighborhood $O(x)$ of $x$ such that

$$
\left\{F_{i}\left(u_{i}, x_{i}^{\prime}, y_{i}^{0}\right): u_{i} \in T_{i}\left(x^{\prime}\right)\right\} \subset-\operatorname{int} C_{i},
$$


whenever $x^{\prime} \in O(x)$, which implies that $O(x) \subset\left\{x \in K: y_{i}^{0} \in S_{i}(x)\right\}$, that is, $\left\{x \in K: y_{i}^{0} \in\right.$ $\left.S_{i}(x)\right\}$ is open. Hence, for each $y_{i} \in P_{i}(x)$, the set $P_{i}^{-1}\left(y_{i}\right)=\left\{x \in K: y_{i} \in \operatorname{int} G_{i}(x) \cap S_{i}(x)\right\}$ is open.

By Lemma 2.4, $\left.P_{i}\right|_{W_{i}}$ has a continuous selection $f_{i}: W_{i} \mapsto 2^{K_{i}}$.

Step 4. We prove that the SGIVQIP has a solution.

For each $i \in I$, define the set-valued map $H_{i}: K \mapsto 2^{K_{i}}$ by

$$
H_{i}(x)= \begin{cases}f_{i}(x), & \text { if } x \in W_{i} \\ G_{i}(x), & \text { if } x \in J_{i}\end{cases}
$$

Note that $H_{i}(x)$ is upper semicontinuous when $x \in \operatorname{int} J_{i}$ and $H_{i}(x)$ is upper semicontinuous when $x \in W_{i}$, and it is easy to verify that $H_{i}(x)$ is also upper semicontinuous when $x \in \partial J_{i}$, where $\partial J_{i}$ denotes the boundary of $J_{i}$. Thus, $H_{i}(x)$ is upper semicontinuous with nonempty convex compact values. By [8, Theorem 7.1.15], the set-valued map $H: K \mapsto 2^{K}$ defined by $H(x)=\prod_{i \in I} H_{i}(x)$ is closed with nonempty convex values. By Kakutani-Fan-Glicksberg's fixed points theorem (see [9, pages 550]), $H$ has a fixed point, that is, there exists $\bar{x} \in H(\bar{x})$. The condition (5) implies that for each $i \in I, \bar{x}_{i} \notin \operatorname{int} G_{i}(\bar{x}) \cap S_{i}(\bar{x})$, that is, $\bar{x}_{i} \neq f_{i}(\bar{x})$ for each $i \in I$. Thus we have that for each $i \in I, \bar{x}_{i} \in G_{i}(\bar{x})$, and

$$
\forall y_{i} \in G_{i}(\bar{x}), \exists \bar{u}_{i} \in T_{i}(\bar{x}), \quad F_{i}\left(\bar{u}_{i}, \bar{x}_{i}, y_{i}\right) \not \subset-\operatorname{int} C_{i} .
$$

The proof is finished.

If $I$ is a singleton, we obtain the following existence result of solutions to the GIVQIP by Theorem 3.2.

Corollary 3.3. Consider a GIVQIP $\{K, D, G, T, F\}$. Assume that

(1) $G(\cdot)$ is continuous on $K$ with convex compact values and for each $x \in K$, int $G(x) \neq \emptyset$;

(2) $T(\cdot)$ is upper semicontinuous on $K$ with nonempty and compact values;

(3) $F(\cdot, \cdot, \cdot)$ is upper $C$-semicontinuous on $D \times K \times K$ with nonempty and compact values;

(4) for each $x \in K$ and each $u \in T(x), F(u, x, \cdot)$ is $C$-convex or C-quasiconvex-like;

(5) for each $x \in K$ and each $u \in T(x)$, if $x \in \operatorname{int} G(x)$, then $F(u, x, x) \not \subset-\operatorname{int} C$.

Then the GIVQIP has a solution, that is, there exists $\bar{x} \in K$ such that $\bar{x} \in G(\bar{x})$,

$$
\forall y \in G(\bar{x}), \exists \bar{u} \in T(\bar{x}), \quad F(\bar{u}, \bar{x}, y) \not \subset-\operatorname{int} C .
$$

Remark 3.4. Theorem 3.2, Corollary 3.3, and each corresponding result in literatures [1-6] do not include each other as a special case.

\section{Acknowledgments}

The research was supported by the Natural Science Foundation of CQ CSTC. 


\section{References}

[1] Y. Chiang, O. Chadli, and J. C. Yao, "Existence of solutions to implicit vector variational inequalities," Journal of Optimization Theory and Applications, vol. 116, no. 2, pp. 251-264, 2003.

[2] P. Cubiotti and J. Yao, "Discontinuous implicit generalized quasi-variational inequalities in Banach spaces," Journal of Global Optimization, vol. 37, no. 2, pp. 263-274, 2007.

[3] L.-J. Lin and H.-L. Chen, "The study of KKM theorems with applications to vector equilibrium problems with implicit vector variational inequalities problems," Journal of Global Optimization, vol. 32, no. 1, pp. 135-157, 2005.

[4] J.-W. Peng, H.-W. J. Lee, and X.-M. Yang, “On system of generalized vector quasi-equilibrium problems with set-valued maps," Journal of Global Optimization, vol. 36, no. 1, pp. 139-158, 2006.

[5] Q. H. Ansari, "Existence of solutions of systems of generalized implicit vector quasi-equilibrium problems," Journal of Mathematical Analysis and Applications, vol. 341, no. 2, pp. 1271-1283, 2008.

[6] S. Al-Homidan, Q. H. Ansari, and S. Schaible, "Existence of solutions of systems of generalized implicit vector variational inequalities," Journal of Optimization Theory and Applications, vol. 134, no. 3, pp. 515531, 2007.

[7] X. Wu and S. Shen, "A further generalization of Yannelis-Prabhakar's continuous selection theorem and its applications," Journal of Mathematical Analysis and Applications, vol. 197, no. 1, pp. 61-74, 1996.

[8] E. Klein and A. C. Thompson, Theory of Correspondences, Canadian Mathematical Society Series of Monographs and Advanced Texts, John Wiley \& Sons, New York, NY, USA, 1984.

[9] C. D. Aliprantis and K. C. Border, Infinite-Dimensional Analysis, Springer, Berlin, Germany, 2nd edition, 1999. 